International Journal of Case Reports
(ISSN:2572-8776)

\title{
Posterior Reversible Encephalopathy Syndrome in Class IV Lupus Nephritis - Case report and brief review of literature
}

\author{
Kalpita Hatti ${ }^{1}$, Amit Habbu ${ }^{2}$, Joe R. Gamboa ${ }^{3}$, Arezoo Haghshenas ${ }^{4}$, Karina D. Torralba ${ }^{5}$
}

${ }^{1}$ Assistant Professor of Medicine, Division of Rheumatology, Department of Medicine, Loma Linda University, Loma Linda, CA; ${ }^{2}$ Attending Radiologist, Kadlec Regional Medical Center, Richland, WA; ${ }^{3}$ Fellow, Division of Rheumatology, Department of Medicine, Loma Linda University, Loma Linda, CA; ${ }^{4}$ Fellow, Division of Rheumatology, Department of Medicine, Loma Linda University, Loma Linda, CA; ${ }^{5}$ Associate Professor of Medicine, Division of Rheumatology, Department of Medicine, Loma Linda University, Loma Linda, CA

\section{ABSTRACT}

Posterior Reversible Encephalopathy Syndrome (PRES) is a ${ }^{*}$ Correspondence to Author: clinico-radiographic syndrome characterized clinically by new on- Dr Kalpita Hatti set neurological manifestations as well as neuroimaging findings 11234 Anderson Street, MCof reversible cerebral edema. We report a case of PRES in the 1516E, Loma Linda, California setting of systemic lupus erythematosus (SLE) with new-onset 92354; Phone: (909)558-4909; Class IV Nephritis treated with pulse corticosteroids, plasma ex- Fax: (909)558-0428. change, intravenous immunoglobulin (IVIG) and cyclophosphamide. We also present a brief review of the associations between PRES and SLE.

How to cite this article:

Kalpita Hatti, Amit Habbu, Joe R. Gamboa, Arezoo Haghshenas, Karina D. Torralba.Posterior Reversible

Keywords: Lupus, Posterior Reversible Encephalopathy SynEncephalopathy Syndrome in Class drome (PRES), encephalopathy

IV Lupus Nephritis - Case report and brief review of literature. International Journal of Case Reports, $20182: 30$

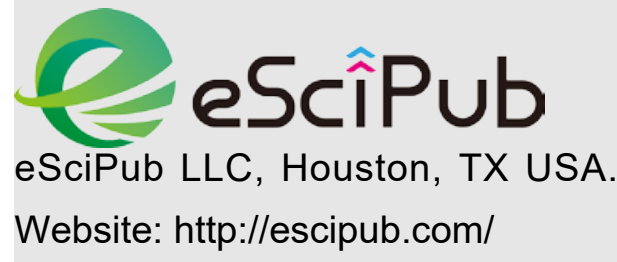




\section{Case Report}

A 29 year old Caucasian female with a twelve year history of SLE (presenting with malar rash, +ANA, +SSA, low C3, low C4, anemia, renal insufficiency with proteinuria/hematuria, arthritis) and a history of class III Lupus Nephritis over the past 3 years presented to the Emergency Room with weakness, lower extremity edema, and worsening proteinuria. Repeat kidney biopsy revealed new onset Class IV Lupus Nephritis. Patient developed acute respiratory failure requiring mechanical ventilation. The patient was treated with intravenous methylprednisolone $1 \mathrm{~g}$ daily for 3 days followed by prednisone $1 \mathrm{mg} / \mathrm{kg}$ daily, plasma exchange with 1 plasma volume every other day for 3 doses followed by intravenous immunoglobulin (IVIG) 2g/kg, and cyclophosphamide IV $(500 \mathrm{mg} / \mathrm{m} 2$ BSA). After one week, she developed altered mental status and had tonic-clonic seizures in the setting of severe hypertension. Brain magnetic resonance imaging (MRI) revealed new onset patchy and confluent areas in the bilateral occipital and posterior temporal lobes (Fig.1) consistent with posterior reversible encephalopathy syndrome (PRES). The patient was treated with antihypertensive (including Clonidine $0.3 \mathrm{mg}$ every 8 hours, metoprolol $100 \mathrm{mg}$ every 8 hours, nifedipine $60 \mathrm{mg}$ every 12 hours and phenytoin 150 mg every 8 hours), resulting in improvement of her neurological symptoms. Patient was discharged home after one week. Patient has continued to do well one year from hospital discharge.

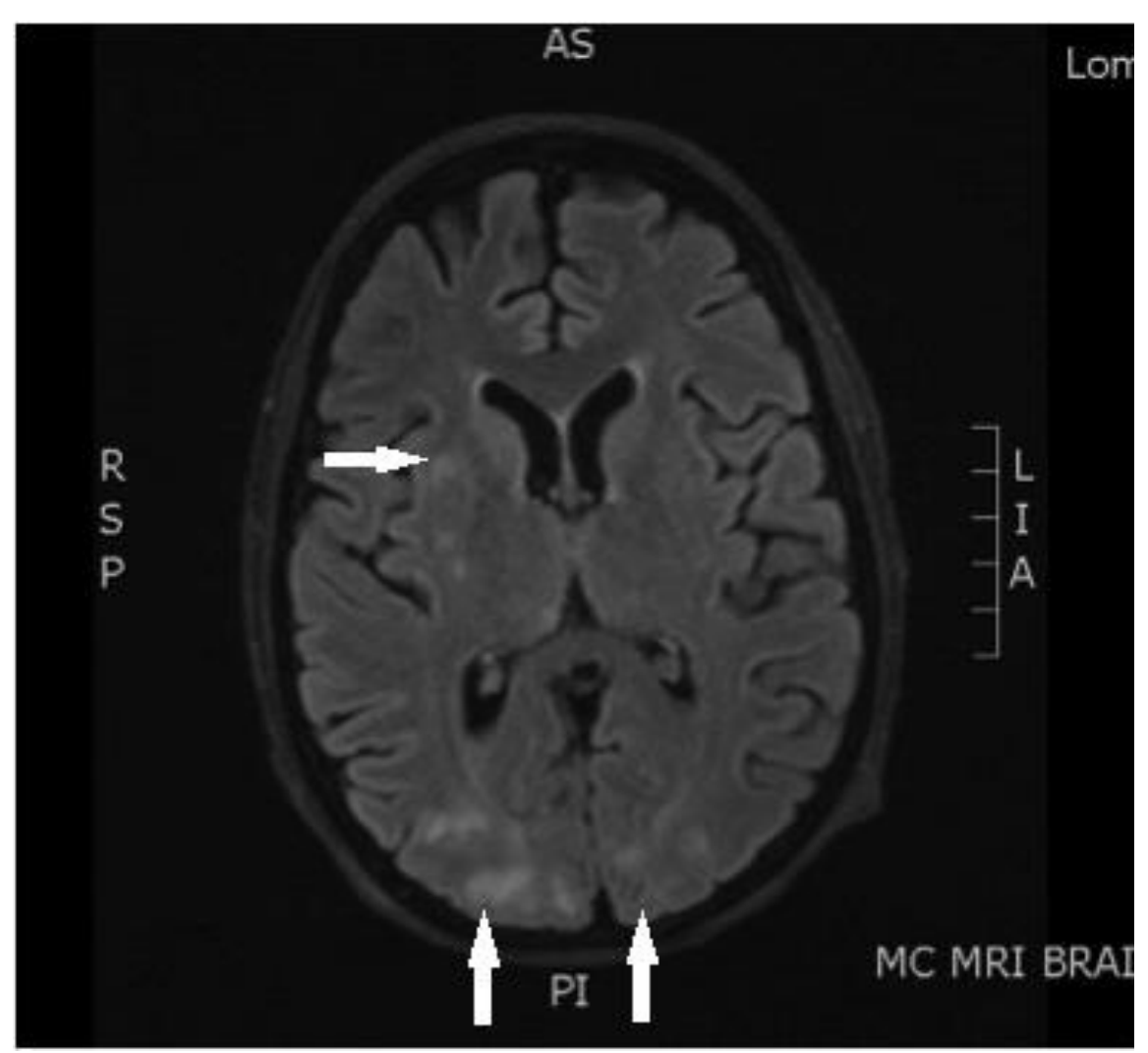

Fig. 1 Axial T2 FLAIR images through the cerebral hemispheres demonstrate areas of edema in the white matter of the occipital lobes bilaterally (vertical arrows) and within the right basal ganglia (horizontal arrow).

\section{Discussion:}

PRES is a clinico-radiographic syndrome that can occur in a subset of SLE patients which presents with a combination of neurological symptoms and corresponding typical brain parenchymal changes best seen on MRI.(1) It 
is also known by the term reversible posterior leukoencephalopathy syndrome (RPLS). The early clinical manifestations of PRES such as headaches, altered mental status, seizures, and visual loss are thought to be associated with white matter vasogenic edema predominantly affecting the posterior occipital and parietal lobes of the cerebral hemispheres.(2) These lupus patients often present with major organ involvement, especially nephritis, necessitating appropriate supportive and curative options including hemodialysis, immunosuppressants, steroids and IVIG. (3) The etiology of PRES in patients with SLE is not yet well established. Cerebral edema caused by endothelial dysfunction is the underlying etiology for PRES, which can be secondary to various triggers such as hypertension, toxic effects of immunosuppressive or cytotoxic drugs, and autoimmune damage in SLE being the most common ones.(7) SLE itself has been proposed as a cause of PRES with triggers such as hypertension or even the use of immunosuppressive agents. Some authors suggest PRES is best considered a secondary complication of SLE or its treatment rather than SLE as a direct cause (4). Given the combined features of neurological symptoms and typical imaging findings in this entity, Mak et al proposed an algorithm for the diagnosis of this condition (5). In a review of 17 SLE cases with PRES, they concluded that urgent brain MRI and initiation of immediate treatment for PRES with very tight blood pressure control and antiepileptic medication are the mainstay of management. Pursuing other etiologies via lumbar puncture and other neurological tests were the alternative pathway in cases with no supporting imaging findings. In 2011, Fujieda et al published their experience with 2 patients and also their meta-analysis.(6) They presented a total of 28 cases of SLE patients with PRES, 15 of which improved with blood pressure control while 13 patients required immunosuppressive therapy for lupus activity resulting in neurological manifestations. Based upon their experience they proposed a treatment algorithm for these patients. All patients who had clinical and supporting imaging findings for PRES were treated promptly with antihypertensive and antiepileptic medications, if a response was achieved the etiology would be "Hypertensive RPLS", otherwise the etiology was assumed as "Immunologic RPLS" and warrant immunosuppressive therapy. (6) Therefore PRES in SLE patients can be categorized into hypertensive or immunological etiologies and treatment should be tailored accordingly in a timely fashion.

Medications such as cyclophosphamide and rituximab, commonly used in SLE, have been reported to predispose patients to PRES. (8) Because it is not unusual for multiple possible etiologies such as hypertension, disease flare, and use of immunosuppressive medication to be concurrently present, a physician's clinical judgment to ascertain the primary cause is vital. (9)

\section{Conclusion}

PRES in SLE is a rare presentation, but can be associated with significant morbidity and mortality if treatment is delayed. Early recognition with neuroimaging is essential. Initial treatment includes immediate blood pressure control and appropriate use of anticonvulsants. In case of suspected triggers such as medications, these must be discontinued if deemed necessary. However in case of non-response to this strategy, an immunological etiology should be entertained and immunosuppressive therapy could be introduced as a treatment.

Conflicts of Interest/Disclosures: None for all authors

\section{Study Support: none}

Each author contributed to this work according to all 4 criteria set by the ICJME, namely 1) substantial contributions to the conception or design of the work; or the acquisition, analysis, 
or interpretation of data for the work; 2) Drafting the work or revising it critically for important intellectual content; 3) Final approval of the version to be published; 4) Agreement to be accountable for all aspects of the work in

\section{References}

1. Ishimori ML, Pressman BD, Wallace DJ, Weisman MH (2007) Posterior reversible encephalopathy syndrome: another manifestation of CNS SLE? Lupus 16, 436-443.

2. Hinchey J, Chaves C, Appignani B, Breen J, Pao L, Wang A, Pessin MS, Lamy C, Mas JL, Caplan LR (1996) A reversible posterior leukoencephalopathy syndrome. $\mathrm{N}$ Engl J Med 334,494-500.

3. Kur JK, Esdaile JM (2006) Posterior reversible encephalopathy syndrome--an underrecognized manifestation of systemic lupus erythematosus. The J Rheumatol 33,2178-2183.

4. Magnano MD, Bush TM, Herrera I, Altman RD (2006) Reversible posterior leukoencephalopathy in patients with systemic lupus erythematosus. Semin Arthritis Rheum 35,396-402.

5. Mak A, Chan BPL, Yeh IB, Ho RCM, Boey ML, Feng PH, Koh DR, Ong BKC (2008) Neuropsychiatric lupus and reversible posterior leucoencephalopathy syndrome: a challenging ensuring that questions related to the accuracy or integrity of any part of the work are appropriately investigated and resolved.

clinical dilemma. Rheumatology 47,256-262.

6. Fujieda $\mathrm{Y}$, Kataoka H, Odani $\mathrm{T}$, Otomo K, Kato M, Fukaya S, Oku K, Horita T, Yasuda S, Atsumi T, Koike T (2011) Clinical features of reversible posterior leukoencephalopathy syndrome in patients with systemic lupus erythematosus. Mod Rheumatol 21,276-281.

7. Mani SH, Shivaprasad SM, Umesh L (2016) A Rare Presentation of Lupus Nephritis Flare up with Posterior Reversible Leucoencephalopathy. 10,OD03-OD04.

8. How J, Blattner M, Fowler S, Wang-Gillam A, Schindler SE (2016) Chemotherapy-associated Posterior Reversible Encephalopathy Syndrome: A Case Report and Review of the Literature. Nephrologist 21,112-117.

9. Zekic T, Benic MS, Antulov R, Antoncic I, Novak $S$ (2017) The multifactorial origin of posterior reversible encephalopathy syndrome in cyclophosphamide-treated lupus patients. Rheumatol Int 37,2105-2114.

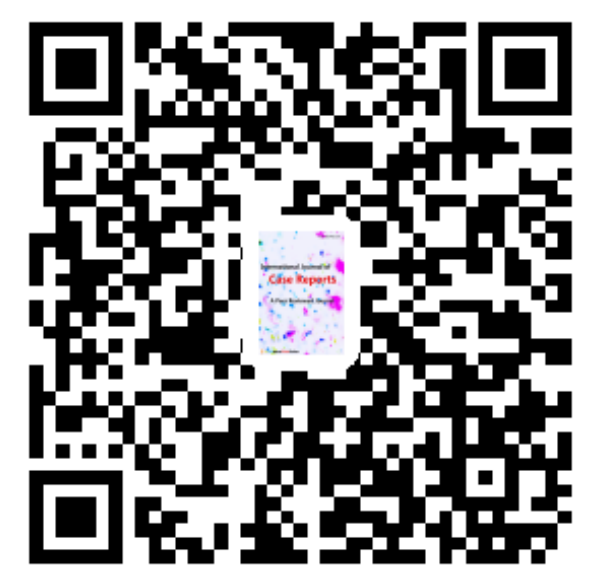

